\title{
MARTIN AND END COMPACTIFICATIONS FOR NONLOCALLY FINITE GRAPHS
}

\author{
DONALD I. CARTWRIGHT, PAOLO M. SOARDI, AND WOLFGANG WOESS
}

\begin{abstract}
We consider a connected graph, having countably infinite vertex set $X$, which is permitted to have vertices of infinite degree. For a transient irreducible transition matrix $P$ corresponding to a nearest neighbor random walk on $X$, we study the associated harmonic functions on $X$ and, in particular, the Martin compactification. We also study the end compactification of the graph. When the graph is a tree, we show that these compactifications coincide; they are a disjoint union of $X$, the set of ends, and the set of improper vertices-new points associated with vertices of infinite degree. Other results proved include a solution of the Dirichlet problem in the context of the end compactification of a general graph. Applications are given to, e.g., the Cayley graph of a free group on infinitely many generators.
\end{abstract}

\section{INTRODUCTION}

In this paper we study transient random walks on infinite graphs, in particular trees, where transitions occur only along the graphs' edges. Our interest is in the positive harmonic functions of the transition matrix in the case when the graph has vertices whose number of neighbors is (countably) infinite. In particular, we want to realize, in terms of the underlying structure, the Martin boundary, i.e., the set of new points in the minimal compactification of the vertex set needed to give an integral representation for every positive harmonic function. Basic references for the definition and construction of the Martin boundary are Doob [Do], Hunt [Hu], Kemeny, Snell, and Knapp [KSK] and Revuz [Re]. There is a slight difference in the definitions of [Do and KSK] on the one hand, and of [Hu and $\mathrm{Re}$ ] on the other. Here, we adopt the latter definition, in which the state space is required to be discrete in the compactification.

While the Martin boundary depends a priori on the transition matrix, we would like to realize it, or at least partially describe it in terms of a "natural" compactification which depends only on the graph structure. This is the end compactification as originally introduced by Freudenthal [Fr] for locally finite graphs. However, in the presence of vertices with infinite degree, the graph theoretical construction of the set of ends (following Halin [Ha]) does not give

Received by the editors March 16, 1990 and, in revised form, May 1, 1991.

1980 Mathematics Subject Classification (1985 Revision). Primary 60J50, 05C05; Secondary $31 \mathrm{C} 20,60 \mathrm{~J} 15$.

Key words and phrases. Martin boundary, ends, harmonic functions on graphs, Fatou theorem, Dirichlet problem.

This research was partially supported by an Australian Research Council grant. 
rise to a compactification of the graph. We show how to modify the construction to obtain an end compactification in the nonlocally finite case: besides ends (equivalence classes of infinite paths), one also has to include what we call improper vertices.

In $\S 2$, after setting up the necessary preliminaries, we introduce the Martin compactification as a typical case of a compactification of the state space (vertex set) with respect to a family of bounded functions, the Martin kernels. We then briefly discuss the general properties of such compactifications.

In $\S 3$, we introduce the family of locally constant functions: they give rise to the end compactification. We then describe this compactification in terms of ends and improper vertices, both for general graphs (Theorem 1) and for trees (Theorem 2). In the tree case, the improper vertices are just new points in one-to-one correspondence with the vertices of infinite degree, and the topology of the compactification may be simply described in terms of geodesics.

In $\S 4$, we compare the Martin and end compactifications. In the tree case they are canonically homeomorphic (Theorem 3), while in the general case there is a canonical map of the Martin compactification onto the end compactification (Proposition 2).

In $\S 5$, we study properties of harmonic functions, showing in the tree case that the minimal Martin boundary is just the set of ends (Proposition 3) and that a nonprobabilistic Fatou theorem holds (Proposition 4). These results generalize to the nonlocally finite case results of Cartier [Ca]. We also solve the Dirichlet problem in the context of the end compactification of a general connected graph (Theorem 4), a new result, we believe, even in the locally finite case.

Finally, in $\S 6$, we explain how these results apply to random walks on infinitely generated free groups, and briefly discuss the difference between the two definitions of Martin boundary mentioned above.

We would like to thank the referee for his careful reading of our manuscript, which resulted in several improvements to the exposition.

\section{Compactifications}

Let $X$ be a countable set of states (later, these will be the vertices of a graph), and let $P$ be a stochastic transition matrix over $X$ with powers $P^{n}, n \geq 0$ $\left(P^{0}=I\right.$, the identity matrix $)$. We assume that $P$ is irreducible and transient; i.e., for all $x, y \in X$, the Green kernel

$$
G(x, y)=\sum_{n=0}^{\infty} P^{n}(x, y)
$$

is strictly positive and finite. If we specify a random variable $Z_{0}$ on $X$, then $P$ gives rise to a Markov chain $\left(Z_{n}\right)_{n \geq 0}$ on $X$ with $\operatorname{Pr}\left[Z_{n+k}=y \mid Z_{k}=x\right]=$ $P^{n}(x, y)$. For given $x, y \in X$, besides $G(x, y)$, the following quantity depends only on $P$ :

$$
H(x, y)=\operatorname{Pr}\left[Z_{n}=y \text { for some } n \geq k \mid Z_{k}=x\right] .
$$

If $h: X \rightarrow \mathbf{R}$, and the sum converges, we define

$$
P h(x)=\sum_{y \in X} P(x, y) h(y),
$$

and call $h$ harmonic if $P h=h$. 
Now fix a base point $o \in X$. The Martin kernel $K=K_{o}: X \times X \rightarrow(0, \infty)$ is given by

$$
K(x, y)=\frac{G(x, y)}{G(o, y)}=\frac{H(x, y)}{H(o, y)} .
$$

A compactification of any (discrete) set $X$ is a compact Hausdorff space $Y$ which contains $X$ as a dense subset and which induces the discrete topology on $X$. Notice that $X$ must be open in $Y$, so that the corresponding boundary $Y \backslash X$ is compact. If $Y$ and $Y^{\prime}$ are two compactifications of $X$, a map $f: Y \rightarrow Y^{\prime}$ is called canonical if it is continuous and $f(x)=x$ for all $x \in X$.

Following [ $\mathrm{Hu}, \mathrm{Re}$ ], we define the Martin compactification of our state space $X$ as the (up to canonical homeomorphism) unique compactification $c_{\mathscr{K}}(X)=$ $c_{\mathscr{K}}(X, P)$ of $X$ with the following properties:

(i) the Martin kernel extends to a continuous function on $X \times c_{\mathscr{K}}(X)$, also denoted $K$, and

(ii) the extended functions $K(x, \cdot), x \in X$, separate the points of the Martin boundary $M=c_{\mathscr{K}}(X) \backslash X$.

The elements of $M$ can be identified with the functions $g$ on $X$ with the following property: there exists a sequence $\left(x_{n}\right)$ of distinct points in $X$ such that $K\left(\cdot, x_{n}\right) \rightarrow g$ pointwise on $X$. The topology on $M$ is then the topology of pointwise convergence. The significance of $M$ rests on the fact that for every positive harmonic function $h$, there is a positive Borel measure $\nu^{h}$ on $M$ such that

$$
h(x)=\int_{M} K(x, \xi) d \nu^{h}(\xi) \text { for } x \in X .
$$

This representation is not always unique. A positive harmonic function $h$ is minimal if, whenever $h \geq h_{1} \geq 0$ for some harmonic function $h_{1}$, then $h_{1} / h$ is constant. The minimal Martin boundary $M_{0}$ is the set of all $\xi \in M$ for which $K(\cdot, \xi)$ is a minimal harmonic function. The representation $(2.1)$ is unique under the additional requirement that $\nu^{h}\left(M \backslash M_{0}\right)=0$.

The construction of the Martin boundary arises as a special case of the following proposition, which is a simplified version of a theorem of Constantinescu and Cornea [CC, §9], also well known in topology (the argument goes back at least to Stone and Cech, see [Ke, pp. 153-154]).

Proposition 1. Let $\mathscr{Q}$ be a family of bounded real valued functions on a set $X$. Then there is an (up to canonical homeomorphism) unique compactification $c_{\mathscr{Q}}(X)$ of $X$ with the following properties:

(I) each $f \in \mathscr{Q}$ extends to a continuous function on $c_{\mathscr{Q}}(X)$, also denoted $f$, and

(II) the extended functions separate the points of $c_{\mathscr{Q}}(X) \backslash X$.

Proof. (Outline) Let $\mathscr{Q}^{*}=\mathscr{Q} \cup\left\{\delta_{x}: x \in X\right\}$, where $\delta_{x}(x)=1$ and $\delta_{x}(y)=0$ if $y \neq x$. For $f \in \mathscr{Q}^{*}$, let $M(f)$ be such that $|f(x)| \leq M(f)$ on $X$. Endow the product space

$$
\prod_{f \in \mathscr{Q}^{*}}[-M(f), M(f)]=\left\{\xi: \mathscr{Q}^{*} \rightarrow \mathbf{R}:|\xi(f)| \leq M(f) \text { for all } f \in \mathscr{Q}^{*}\right\}
$$

with the product topology, and embed $X$ by the mapping $x \mapsto \xi_{x}$, where $\xi_{x}(f)=f(x)$ for $f \in \mathscr{Q}^{*}$. This mapping is injective, since $\delta_{x} \in \mathscr{Q}^{*}$ for each 
$x \in X$. Identify $X$ with its image. The required compactification is the closure of $X$ in the product space, and we extend $f \in \mathscr{Q}$ by writing $f(\xi)=\xi(f)$ for $\xi$ in the closure. It is clear that (I) and (II) hold, and straightforward (see [CC]) to check uniqueness using the following description of convergence of nets:

$$
\text { A net }\left(x_{\alpha}\right) \text { in } c_{\mathscr{Q}}(X) \text { converges to a point in } c_{\mathscr{Q}}(X) \backslash X \text { if and only if }
$$

- for every finite $U \subset X$ there is an $\alpha_{0}$ such that $x_{\alpha} \notin U$ for every $\alpha>\alpha_{0}$, and

- $\lim _{\alpha} f\left(x_{\alpha}\right)$ exists for every $f \in \mathscr{Q}$.

Alternatively, $c_{\mathscr{Q}}(X)$ can be characterised by the following minimality property:

Lemma 1. Let $Y$ be a compactification of $X$ such that each $f \in \mathscr{Q}$ extends to a continuous function on $Y$. Then there is a canonical map of $Y$ onto $c_{\mathscr{Q}}(X)$.

Proof. Use the above description of convergence of nets.

If $\mathscr{Q}_{1}$ and $\mathscr{Q}_{2}$ are two families of bounded functions on $X$, with $\mathscr{Q}_{1} \subset \mathscr{Q}_{2}$, then there is a canonical map of $c_{\mathscr{Q}_{2}}(X)$ onto $c_{\mathscr{Q}_{1}}(X)$. If $\mathscr{Q}_{0} \subset \mathscr{Q}$, and each $f \in \mathscr{Q}$ is a finite linear combination of functions in $\mathscr{Q}_{0}$, then $c_{\mathscr{Q}}(X)$ and $c_{\mathscr{Q}_{0}}(X)$ are canonically homeomorphic. In particular, if $X$ and $\mathscr{Q}_{0}$ are countable, then $c_{\mathscr{Q}}(X)$ is metrizable. These facts are simple consequences of the last lemma. Note that any compactification of $X$ is $c_{\mathscr{Q}}(X)$ for some $\mathscr{Q}$.

In particular, consider the family $\mathscr{K}=\{K(x, \cdot): x \in X\}$. Each $f \in \mathscr{K}$ is bounded, since $0<K(x, y) \leq 1 / H(o, x)$. Thus taking $\mathscr{Q}=\mathscr{K}$, Proposition 1 provides a construction of the Martin boundary.

\section{LOCALLY CONSTANT FUNCTIONS}

In this section we assume that $X$ is the countable vertex set of an infinite connected graph $\Gamma$, whose edge set we denote by $E$ (the edges are unoriented, we allow loops, but no multiple edges). We write $d(x, y)$ for the usual distance in $\Gamma$. A locally constant function is a real-valued function $f$ on $X$ for which $\{[x, y] \in E: f(x) \neq f(y)\}$ is finite. The linear space of locally constant functions is denoted by $\mathscr{L}$. We denote by $\mathscr{F}(Y)$ the family of finite subsets of a set $Y$. If $F \in \mathscr{F}(E)$, write $\Gamma \backslash F$ for the graph with vertex set $X$ and edge set $E \backslash F$. We also let $\delta F$ denote the set of vertices which are incident with some edge in $F$. Now let $\mathscr{L}_{0}$ be the subfamily of $\mathscr{L}$ consisting of all functions $f=\chi_{C}$, where $C$ is a connected component of $\Gamma \backslash F$ for some $F \in \mathscr{F}(E)$. It is clear that $\mathscr{L}_{0}$ is countable and spans $\mathscr{L}$. The end compactification of $\Gamma$ is defined to be the space $c_{\mathscr{L}}(X)=c_{\mathscr{L}_{0}}(X)$, according to Proposition 1. This compactification is metrizable, and so we may restrict ourselves to sequences instead of nets in our description (2.2) of convergence to points of $\partial \Gamma=c_{\mathscr{L}}(X) \backslash X$.

Lemma 2. A sequence $\left(x_{n}\right)$ of distinct elements of $X$ converges to a point $\xi$ of $\partial \Gamma$ if and only if for each $F \in \mathscr{F}(E)$ there is a component $C_{F}$ of $\Gamma \backslash F$ which contains all but finitely many of the $x_{n}$. The resulting family $\left\{C_{F}: F \in \mathscr{F}(E)\right\}$ depends only on $\xi$ and not on the particular sequence $\left(x_{n}\right)$ converging to it, and the application $\xi \mapsto\left\{C_{F}: F \in \mathscr{F}(E)\right\}$ is injective.

Proof. If $\left(x_{n}\right)$ converges, then we must have

$$
\lim \chi_{C}\left(x_{n}\right)=0 \quad \text { or } \quad \lim \chi_{C}\left(x_{n}\right)=1
$$


for each component $C$ of $\Gamma \backslash F$, whenever $F \in \mathscr{F}(E)$. Hence, there must be precisely one component $C_{F}$ for which the limit is 1 . Conversely, if the $C_{F}$ exist as proposed, then (3.1) holds for each $\chi_{C} \in \mathscr{L}_{0}$, and so $\left(x_{n}\right)$ must converge to a $\xi \in \partial F$. Furthermore, for the continuous extension of $\chi_{C_{F}}$ we must have $\chi_{C_{F}}(\xi)=1$, and this determines $C_{F}$ among the components of $\Gamma \backslash F$. Hence $\left(C_{F}\right)$ is independent of $\left(x_{n}\right)$. The last statement follows from the separation property (II).

In what follows, when speaking of a family of components $\left(C_{F}\right)$, we assume that $F$ varies over $\mathscr{F}(E)$ and each $C_{F}$ is one of the finitely many components of $\Gamma \backslash F$. In the situation of Lemma 2, we say that $\left(C_{F}\right)$ determines $\xi$.

Lemma 3. A family of components $\left(C_{F}\right)$ determines an element of $\partial \Gamma$ if and only if

(a) each $C_{F}$ is infinite, and

(b) $C_{F_{2}} \subset C_{F_{1}}$ whenever $F_{1} \subset F_{2}$.

Proof. If $\left(C_{F}\right)$ determines an element of $\partial \Gamma$, then it follows from Lemma 2 that (a) and (b) hold. Conversely, let $\left(F_{n}\right)_{n \geq 0}$ be a sequence in $\mathscr{F}(E)$ such that $F_{n} \subset F_{n+1}$ for all $n \geq 0$ and $\bigcup_{n \geq 0} F_{n}=E$. By (a) and (b), we can find a sequence $\left(x_{n}\right)$ of distinct elements of $X$ such that $x_{n} \in C_{F_{n}}$ for each $n$. If $F \in \mathscr{F}(E)$, then there is a $k$ such that $F \subset F_{k}$, and so $C_{F_{n}} \subset C_{F_{k}} \subset C_{F}$ for all $n \geq k$. Hence $x_{n} \in C_{F}$ for all $n \geq k$, so that $\left(x_{n}\right)$ converges to an element of $\partial \Gamma$ determined by $\left(C_{F}\right)$.

Remark 1. Lemmas 2 and 3 say that $\partial \Gamma$ can be described as an inverse limit. For every $f \in \mathscr{F}(E)$ let $\mathscr{C}_{F}$ denote the set of infinite components of $\Gamma \backslash F$. With the discrete topology, $\mathscr{C}_{F}$, being finite, is compact. If $F_{1} \subset F_{2}, F_{2} \in$ $\mathscr{F}(E)$, then every $C \in \mathscr{C}_{F_{2}}$ is contained in an element of $\mathscr{C}_{F_{1}}$, denoted $\pi_{F_{2}, F_{1}}(C)$. As $\mathscr{F}(E)$ is directed with respect to set inclusion, the maps $\pi_{F_{2}, F_{1}}$ : $\mathscr{C}_{F_{2}} \rightarrow \mathscr{C}_{F_{1}}$ give rise to an inverse limit of the system $\left\{\mathscr{C}_{F}: F \in \mathscr{F}(E)\right\}$. Using Lemmas 2 and 3, one obtains that it coincides with $\partial \Gamma$.

In the locally finite case, $c_{\mathscr{L}}(X)$ is the inverse limit of the family $\left\{\mathscr{C}_{F}^{\prime}: F \in\right.$ $\mathscr{F}(E)\}$, where $\mathscr{C}_{F}^{\prime}$ consists of all components of $\Gamma \backslash F$, but in the general case the natural map of $X$ into this inverse limit need not even be injective.

We now give a graph theoretical description of $\partial \Gamma$ in the setting of a general graph, extending the approach of [Ha], cf. also Jung [Ju]. Also, compare our approach with the rather abstract topological one of Polat [Po], where, however, finite sets of vertices play the same role as finite sets of edges here.

Let $X^{\infty}$ be the set of vertices of infinite degree. An infinite path or ray is a sequence $\left[x_{0}, x_{1}, x_{2}, \ldots\right]$ of distinct vertices with $x_{i}$ and $x_{i+1}$ adjacent for each $i \geq 0$. We denote by $\Pi$ the set of infinite paths. Let $F \in \mathscr{F}(E)$. If $x \in X^{\infty}$, then $x \in C_{F}$ for an infinite component $C_{F}=C_{F}(x)$ of $\Gamma \backslash F$. If $\pi=\left[x_{0}, x_{1}, \ldots\right] \in \Pi$, then an infinite component $C_{F}(\pi)$ of $\Gamma \backslash F$ must contain all but finitely many of the $x_{n}$. We now define an equivalence relation on $X^{\infty} \cup \Pi$ : If $z_{1}, z_{2} \in X^{\infty} \cup \Pi$, then $z_{1} \sim z_{2}$ if and only if $C_{F}\left(z_{1}\right)=C_{F}\left(z_{2}\right)$ for each $F \in \mathscr{F}(E)$. If $\xi=[z] \in\left(X^{\infty} \cup \Pi\right) / \sim$, we write $C_{F}(\xi)=C_{F}(z)$. We add to each $C_{F}$ all $\xi$ with $C_{F}(\xi)=C_{F}$. An end is an equivalence class in $\left(X^{\infty} \cup \Pi\right) / \sim$ which contains some infinite path. The set of ends is denoted by $\Omega$. An equivalence class which consists of elements of $X^{\infty}$ only is called an improper vertex. The set of improper vertices is denoted $X^{*}$. 
We remark that our definition of ends agrees with the one of [Ha], who calls two infinite paths equivalent if there is a third which meets each of the other two infinitely often. The only difference is that our equivalence classes may also contain elements of $X^{\infty}$. The improper vertices, however, are new, and serve to obtain the desired compactification, compare with [Po].

Theorem 1. We may identify $\partial \Gamma$ with $\Omega \cup X^{*}$. In the topology of $c_{\mathscr{L}}(X), a$ neighborhood base of $\xi \in \Omega \cup X^{*}$ is given by

$$
\left\{C_{F}(\xi) \backslash U: F \in \mathscr{F}(E), \quad U \in \mathscr{F}(X)\right\} .
$$

Proof. If $\xi \in \Omega \cup X^{*}$, then it is clear that $\left(C_{F}(\xi)\right)$ satisfies (a) and (b) of Lemma 3. By definition of the equivalence relation, different elements of $\Omega \cup X^{*}$ give rise to different families $\left(C_{F}\right)$. Thus, $\Omega \cup X^{*}$ naturally embeds into $\partial \Gamma$. We prove that this embedding is onto.

Let $\left(C_{F}\right)$ be a family of components with properties (a) and (b) of Lemma 3. Assume that $\left(C_{F}\right) \neq\left(C_{F}(x)\right)$ for all $x \in X^{\infty}$. We have to construct an infinite path $\pi$ such that $\left(C_{F}\right)=\left(C_{F}(\pi)\right)$. We claim that, given $x_{0} \in X$, there is a sequence $\left(F_{n}\right)$ in $\mathscr{F}(E)$ such that

$$
x_{0} \notin C_{F_{1}} \text { and for } n \geq 2 F_{n-1} \subset F_{n} \text { and } \delta F_{n-1} \cap C_{F_{n}}=\varnothing \text {. }
$$

Indeed, if $x_{0} \in X^{\infty}$, then by the assumption that $\left(C_{F}\right) \neq\left(C_{F}(x)\right)$ for all $x \in X^{\infty}, x_{0} \notin C_{F_{1}}$ for some $F_{1} \in \mathscr{F}(E)$. If $x_{0}$ has finite degree, then let $F_{1}$ be the set of edges incident with $x_{0}$. As $C_{F_{1}}$ is infinite by (a), we must have $x_{0} \notin C_{F_{1}}$. Now assume that we have already constructed $F_{1}, \ldots, F_{n-1}$. As above, for every $y \in \delta F_{n-1}$ we must have $y \notin C_{F(y)}$ for some $F(y) \in \mathscr{F}(E)$. Let $F_{n}$ be the union of $F_{n-1}$ and all these $F(y)$.

Now let $\pi_{1}$ be a shortest path from $x_{0}$ to some element in $C_{F_{1}}$ with endpoint $x_{1}$. Then $x_{1} \in \delta F_{1}$ and $\pi_{1} \cap C_{F_{1}}=\left\{x_{1}\right\}$. Once more, we proceed by induction: assume we have already extended $\pi_{1}$ to a finite path $\pi_{n-1}$ without repetitions, whose endpoint $x_{n-1}$ lies in $\delta F_{n-1}$, such that $\pi_{n-1} \cap C_{F_{n-1}}=\left\{x_{n-1}\right\}$. Then, as $C_{F_{n-1}}$ is connected, we can find a shortest path in $C_{F_{n-1}}$ which connects $x_{n-1}$ to some point of $C_{F_{n}}$. If we use this path to extend $\pi_{n-1}$, we obtain $\pi_{n}$ with endpoint $x_{n}$ to conclude the induction. At each step, the length of $\pi_{n}$ increases at least by one because of the last condition in (3.2), so that in the limit, we obtain an infinite path $\pi$ such that $C_{F_{n}}=C_{F_{n}}(\pi)$ for each $n$.

If $x \in X$, then $d\left(x, C_{F_{n}}\right) \rightarrow \infty$. For $d\left(x, C_{F_{n}}\right) \geq d\left(x_{0}, C_{F_{n}}\right)-d\left(x, x_{0}\right)$, and any path from $x_{0}$ to a point of $C_{F_{n}}$ has length at least $n$. Thus if $F \in$ $\mathscr{F}(E)$ is arbitrary, we can find $n$ such that $\delta F \cap C_{F_{n}}=\varnothing$. Therefore $C_{F_{n}}$ is connected in $\Gamma \backslash F$, and so either $C_{F_{n}} \cap C_{F}=\varnothing$ or $C_{F_{n}} \subset C_{F}$. By Lemma 3(b), the former is impossible. Thus $C_{F_{n}} \subset C_{F}$. Hence $C_{F}=C_{F}(\pi)$. In other words, if $\omega \in \Omega$ is the equivalence class of $\pi$, then $C_{F}=C_{F}(\omega)$ for every $F \in \mathscr{F}(E)$ : the embedding is onto.

Finally, the neighborhood base is obtained by reformulating the product topology of $c_{\mathscr{L}_{0}}(X)$. Observe that the finite set $U \subset X$ are indispensable if $X^{\infty} \neq \varnothing$. Indeed, if $x \in X^{\infty}$ and $x^{*}=[x]$ is the associated equivalence class in $\partial \Gamma$, then $\bigcap_{F \in \mathscr{F}(E)} C_{F}\left(x^{*}\right) \supset\left\{x, x^{*}\right\}$.

We remark that it is not hard to see that the path $\pi$ constructed in the above proof is an infinite geodesic, i.e., each of its finite subpaths is shortest. Thus, we may also describe the end compactification in terms of equivalence classes of infinite degree vertices and infinite geodesics. 
In the case when $\Gamma$ is a (nonlocally finite, countably infinite) tree, we can describe $X^{*}$ and $\Omega$ rather more explicitly. Recall that the geodesic between two vertices $x, y \in X$ is the unique shortest path in $X$ connecting $x$ and $y$. An infinite path $\pi=\left[x_{0}, x_{1}, \ldots\right]$ as defined above is just an infinite geodesic in this case, i.e., $\left[x_{m}, \ldots, x_{n}\right]$ is the geodesic from $x_{m}$ to $x_{n}$ for each $m \leq n$. If $x \in X^{\infty}$, the set of vertices of infinite degree, and if $\pi \in \Pi$, the set of infinite paths, there is an $F \in \mathscr{F}(E)$ such that $C_{F}(x) \neq C_{F}(\pi)$, so $x$ and $\pi$ cannot be equivalent. Two infinite paths are equivalent if and only if they differ only by finitely many vertices. As before, an equivalence class of a $\pi \in \Pi$ is called an end and the set of all ends is denoted $\Omega$. If $x \in X$ and $\omega \in \Omega$, then there is presicely one ray which representes $\omega$ and emanates from $x_{0}=x$. This is the geodesic from $x$ to $\omega$. Thus we may think of $\Omega$ as the set of rays emanating from a fixed base point $o \in X$.

If $x \in X^{\infty}$, we denote by $x^{*}$ the equivalence class of $x$. Thus $x^{*}$ is an improper vertex as defined above. The tree structure shows that $x^{*} \neq y^{*}$ if $x, y \in X^{\infty}$ are distinct. The set $X^{*}$ with elements $x^{*}, x \in X^{\infty}$, is disjoint from $X$ and $\Omega$. We may imagine $x^{*}$ as "sitting above" $x$, linked by $x$ by an improper edge. In this way we obtain an enlarged tree, with vertex set $X \cup X^{*}$.

If $z, w \in X \cup \Omega$, then the confluent of $z$ and $w$ with respect to $o \in X$, denoted $\langle z, w\rangle_{o}$, is the last common vertex on the geodesics from $o$ to $z$ and from $o$ to $w$ respectively. If $z=w=\omega \in \Omega$, there is no such vertex, and we write $\langle\omega, \omega\rangle_{0}=\omega$. We extend this notion to $X^{*}$ by defining $\left\langle x^{*}, z\right\rangle_{o}=$ $\langle x, z\rangle_{o}$ and $\left\langle x^{*}, y^{*}\right\rangle_{o}=\langle x, y\rangle_{o}$ if $x, y \in X^{\infty}$ and $z \in X \cup \Omega$.

We now introduce a topology on $\widetilde{X}=X \cup X^{*} \cup \Omega$ so that

- $\{x\}$ is open for every $x \in X$.

- If $\omega \in \Omega$, then a neighborhood base at $\omega$ is given by the sets $B_{x}(\omega)=$ $\left\{z \in \tilde{X}:\langle z, x\rangle_{o}=x\right\}$, where $x$ varies on the geodesic from $o$ to $\omega$.

- If $x^{*} \in X^{*}$, then a neighborhood base at $x^{*}$ is given by the sets $B_{U}\left(x^{*}\right)=$ $\left\{z \in \tilde{X}: z \neq x,\langle z, y\rangle_{x}=x\right.$ for every $\left.y \in U\right\}$, where $U$ varies among finite sets of neighbors of $x$.

A sequence $\left(x_{n}\right)$ in $X$ converges in this topology to $\omega \in \Omega$ if and only if the distance between $o$ and $\left\langle x_{n}, \omega\right\rangle_{o}$ tends to infinity; it converges to $y^{*} \in X^{*}$ if and only if the geodesics from $y$ to $x_{n}$ start with any given edge for only finitely many $n$.

Theorem 2. For a tree $\Gamma, c_{\mathscr{L}}(X)$ is homeomorphic to $\tilde{X}$ with the topology described above, and $\partial \Gamma=X^{*} \cup \Omega$.

Proof. By Theorem 1 and the above discussion, we may identify $c_{\mathscr{L}}(X)$ and $\tilde{X}$. It is easy to check that this identification is a homeomorphism. Suppose, for example, that $x \in X^{\infty}$. A base of neighborhoods at $x^{*} \in X^{*}$ in $c_{\mathscr{L}}(X)$ consists of sets $\overline{C_{F}(x)} \backslash S$, where $F \in \mathscr{F}(E)$ and $S \subset X$ is finite. Suppose that $\left(\xi_{n}\right)$ is a sequence in $\tilde{X}$ converging to $x^{*}$ there. Given $F$ and $S$, let $U$ be the set of neighbors of $x$ lying on geodesics from $x$ to vertices in $\delta F \cup S$. Then $\xi_{n} \in \overline{C_{F}(x)} \backslash S$ once $\xi_{n} \in B_{U}\left(x^{*}\right)$.

\section{COMPARING MARTIN AND END COMPACTIFICATIONS}

In this section, we assume that $\Gamma$ is an infinite connected graph with countable vertex set $X$, and that $P$ is a transient transition matrix over $X$ which is 
adapted to the graph structure as follows:

$$
P(x, y)>0 \text { if and only if }[x, y] \in E .
$$

Note in particular that $P(y, x)>0$ if $P(x, y)>0$.

Let us first consider the case when $\Gamma$ is a tree. In [Ca], this has been studied in full detail in the locally finite case, and many of the results there carry over directly. In particular, the following remains valid without changes:

Lemma 4. If $\Gamma$ is a tree, and if $x, y \in X$, then

(a) if $v$ lies on the geodesic from $x$ to $y$, then $H(x, y)=H(x, v) H(v, y)$;

(b) The Martin kernel $K=K_{o}$ satisfies $K(x, y)=K\left(x,\langle x, y\rangle_{o}\right)=$ $K\left(x,\langle x, o\rangle_{y}\right)$.

Theorem 3. For a tree $\Gamma$, we have $\mathscr{K} \subset \mathscr{L}$, and there is a canonical homeomorphism between the end compactification $c_{\mathscr{L}}(X)$ and the Martin compactification $c_{\mathscr{H}}(X)$.

Proof. If $x \in X$, then by Lemma 4(b), the only edges $[v, y]$ where $K(x, v)$ and $K(x, y)$ may differ are those of the geodesic from $o$ to $x$. Hence $K(x, \cdot) \in \mathscr{L}$, so that $\mathscr{K} \subset \mathscr{L}$. By Lemma 1 , there is a canonical map of $c_{\mathscr{L}}(X)$ onto $c_{\mathscr{H}}(X)$. Indeed, the continuous extension of $K(x, \cdot)$ to $c_{\mathscr{L}}(X)=$ $\widetilde{X}$ is given by

$$
K(x, \xi)=K\left(x,\langle x, \xi\rangle_{o}\right) .
$$

It is easy to check continuity using the description of convergence preceding Theorem 2.

In order to prove that the canonical mapping is a homeomorphism, we have to verify the following: if $\xi, \eta \in \partial \Gamma$ and $K(\cdot, \xi)=K(\cdot, \eta)$, then $\xi=\eta$. Checking this amounts to showing that the numbers $K(x, y)$, where $y$ varies along the geodesic from $o$ to $x$, are distinct. This follows from Lemma 4(a) and transience, which implies that $H(u, v) H(v, u)<1$ for $u, v \in X$ distinct.

The last part of the above proof could have been replaced by appealing to the next proposition, adapted from [PW2]. We remark that even in the case of an isotropic random walk in a locally finite homogeneous tree, the Martin boundary may fiber over the end boundary if $P(x, y)>0$ is allowed for unbounded $d(x, y)$ (see the recent paper of Cartwright and Sawyer [CS]).

Proposition 2. Let $\Gamma$ be an arbitrary connected graph with countable vertex set. If $P$ is transient and satisfies (4.1), then there is a canonical continuous map of the Martin compactification $c_{\mathscr{H}}(X)$ onto the end compactification $c_{\mathscr{L}}(X)$.

Proof. In view of (2.2), and the compactness and metrizability of $c_{\mathscr{H}}(X)$ and $c_{\mathscr{L}}(X)$, we have to prove the following: if $\left(x_{n}\right)$ and $\left(y_{n}\right)$ are two sequences in $X$ such that

(1) $\lim K\left(\cdot, x_{n}\right)=\lim K\left(\cdot, y_{n}\right)=f$ exists pointwise, and

(2) $x_{n} \rightarrow \xi$ and $y_{n} \rightarrow \eta$ in the end compactification, where $\xi, \eta \in \partial \Gamma$, then $\xi=\eta$ must hold. Assume the contrary. The $C_{F}(\xi) \neq C_{F}(\eta)$ for some $F \in \mathscr{F}(E)$. Let $U=U(\xi)$ denote the set of $x \in \delta F \cap C_{F}(\xi)$ for which there is some finite path from $C_{F}(\eta)$ which enters $C_{F}(\xi)$ at $x$. Define a set $V=V(\eta)$ similarly, but with the roles of $\xi$ and $\eta$ interchanged. Note that $U \cap V=\varnothing$, and that both sets are finite. 
For $x \in U$ and $y \in V$, define

$$
a(x, y)=\operatorname{Pr}\left[Z_{n} \text { enters } C_{F}(\eta) \text { at } y \mid Z_{0}=x\right],
$$

and

$$
b(y, x)=\operatorname{Pr}\left[Z_{n} \text { enters } C_{F}(\xi) \text { at } x \mid Z_{0}=y\right] .
$$

By (4.1) and the definition of $U$ and $V$, the matrices $A=(a(x, y))_{x \in U, y \in V}$ and $B=(b(y, x))_{y \in V, u \in U}$ have strictly positive entries and are substochastic. Starting at $x \in U$, the random walk must pass through $V$ before entering $C_{F}(\eta)$. Now $y_{n} \in C_{F}(\eta)$ for all but finitely many $n$. Hence, for $n$ sufficiently large,

$$
H\left(x, y_{n}\right)=\sum_{y \in V} a(x, y) H\left(y, y_{n}\right) .
$$

If we divide by $H\left(o, y_{n}\right)$ and let $n$ tend to infinity, we obtain

$$
f(x)=\sum_{y \in V} a(x, y) f(y) \text { for every } x \in U \text {. }
$$

We similarly obtain

$$
f(y)=\sum_{x \in U} b(y, x) f(x) \text { for every } y \in V .
$$

Combining the last two identities, we see that the substochastic square matrix $A B$ has 1 as an eigenvalue. Since all its entries are strictly positive, it must be strictly stochastic. Hence, also $A$ and $B$ must be stochastic in each row. Thus, starting from some point in $U$, the random walk visits $V$ with probability one, and vice versa. As a consequence, $Z_{n}$ returns to the finite set $U$ infinitely often with probability one. This contradicts transience.

We remark that, contrary to the locally finite case [PW2], Proposition 2 may not be obtained as an application of the abstract result of Taylor [Ta, Corollary 4 , p. 449]. The reason is that $\Gamma$ does not give rise to a locally compact space, if we view it as a one-dimensional polygonal complex where each edge is a copy of the unit interval.

\section{Properties OF HARMONIC FUNCTIONS}

The main object of this section is the study of the boundary behavior of harmonic functions. We always assume that $P$ is transient and satisfies (4.1). As in the preceding sections, we start with results concerning trees.

Proposition 3. If $\Gamma$ is a tree, then the minimal Martin boundary is $\Omega$.

Proof. First of all, if $x^{*} \in X^{*}$, then $K\left(\cdot, x^{*}\right)=K(\cdot, x)$ which is not harmonic. Thus, $x^{*} \notin M_{0}$.

Next, we verify that $K(\cdot, \omega)$ is harmonic for $\omega \in \Omega$. Let $x \in X$, and let $u$ be the next vertex after $\langle x, \omega\rangle_{o}$ on the geodesic from $o$ to $\omega$. By (4.2) and Lemma $4, K(y, \omega)=K(y, u)$ if $y$ is $x$ or one of its neighbors. Hence,

$$
\sum_{y} P(x, y) K(y, \omega)=\sum_{y} P(x, y) K(y, u)=K(x, u)=K(x, \omega) .
$$

Finally, it is well known that $K(\cdot, \omega)$ is minimal if we cannot write

$$
K(\cdot, \omega)=t h_{1}+(1-t) h_{2}, \quad 0<t<1,
$$


where $h_{1}, h_{2}$ are two different positive harmonic functions with $h_{i}(o)=1$. Assume that (5.1) holds. Observe that $h(x) \geq H(x, y) h(y)$ for all $x, y \in X$, if $h$ is positive harmonic. Indeed, $G_{h}(x, y)=G(x, y) h(y) / h(x)$ is the Green kernel of the transient transition matrix $P_{h}(x, y)=P(x, y) h(y) / h(x)$, and therefore satisfies $G_{h}(x, y) \leq G_{h}(y, y)$.

By (4.2) and Lemma 4, $K(x, \omega)=H(x, y) K(y, \omega)$, if $y$ lies on the geodesic from $x$ to $\omega$. Hence,

$$
\begin{aligned}
t h_{1}(x)+(1-t) h_{2}(x) & =H(x, y) K(y, \omega) \\
& =t H(x, y) h_{1}(y)+(1-t) H(x, y) h_{2}(y),
\end{aligned}
$$

and we must have $h_{i}(x)=H(x, y) h_{i}(y)$ whenever $y$ lies on the geodesic from $x$ to $\omega$. In particular, choose $y=\langle x, \omega\rangle_{o}$. Since $1=h_{i}(o)=H(o, y) h_{i}(y)$, we obtain, using (4.2),

$$
h_{i}(x)=h_{i}(x) / h_{i}(o)=K(x, y)=K(x, \omega) .
$$

This yields minimality of $\omega$.

We remark that the last part of the above proof has been adapted from Derriennic [De].

Let us state the Fatou theorem for the tree $\Gamma$. A function $g: X \rightarrow[0, \infty)$ is called superharmonic if $P g \leq g$. If $h$ is a positive harmonic function on $X$, then any such $g$ can be written

$$
g(x)=\sum_{y \in X} G(x, y) v(y)+\int_{M} K(x, \cdot) \varphi d \nu^{h}+h^{\prime}(x),
$$

where $v, h^{\prime}: X \rightarrow[0, \infty), M$ is the Martin boundary, $\varphi: M \rightarrow[0, \infty)$ is $\nu^{h_{-}}$ integrable, and $h^{\prime}$ is harmonic, with $\nu^{h^{\prime}}$ singular with respect to $\nu^{h}$ (see [Ca, Proposition 3.2]).

Proposition 4. With the above notation, for $\nu^{h}$-almost all $\omega \in \Omega, g(x) / h(x)$ tends to $\varphi(\omega)$ as $x \in X$ tends to $\omega$ along the geodesic from $o$ to $\omega$.

Proof. Cartier [Ca, Théorème 3.3] proved this in the locally finite case. His proof remains valid in our case: one need only remark that his Théorème 1.2, which he uses there, is still true. To see this requires a proof that if $\left(t_{0}, t_{1}, \ldots\right)$ is a sequence of vertices such that $t_{i}$ and $t_{i+1}$ are adjacent for each $i \geq 0$, and such that for each $t \in X$, the set $\left\{j \geq 0: t_{j}=t\right\}$ is finite, then $d\left(t_{0}, t_{j}\right) \rightarrow$ $\infty$. So let $J_{n}=\left\{j \geq 0: d\left(t_{0}, t_{j}\right)=n\right\}$. Then $J_{0}$ is finite by hypothesis. Assuming that $J_{n}$ is finite, $J_{n+1}$ must be finite too. For otherwise there must be infinitely many distinct $t_{j}$ 's at distance $n+1$ from $t_{0}$. But between any two corresponding $j$ 's there must be a $k \in J_{n}$, a contradiction. So each $J_{n}$ is finite and the assertion is proved.

In the last theorem, we return to general graphs $\Gamma$. Given a compactification $c_{\mathscr{Q}}(X)$, we can state the Dirichlet problem: does every continuous real function on the boundary $c_{\mathscr{Q}}(X) \backslash X$ have a continuous extension to $c_{\mathscr{Q}}(X)$ which is harmonic on $X$ ? We remark that, due to the Maximum Principle, such a solution must be unique if it exists. We now show that the answer is positive for the end compactification under an additional assumption. We say that the Green kernel vanishes at infinity, if for every $\varepsilon>0$, the set $\{x \in X: G(x, o) \geq \varepsilon\}$ is finite. 
Theorem 4. Assume that $P$, given on the graph $\Gamma$, satisfies (4.1), and that the Green kernel vanishes at infinity. Then the Dirichlet problem is solvable with respect to the end compactification.

Proof. In terms of the random walk, transience means that $Z_{n}$, starting at $Z_{0}=x \in X$, almost surely leaves every finite set after a finite time. This applies, in particular, to the sets $\delta F$, where $F \in \mathscr{F}(E)$. Thus, with probability one, $Z_{n}$ ends up in one of the components of $\Gamma \backslash F$. In view of the countable base of the topology on $c_{\mathscr{L}}(X)$ described in Theorem 1 , this implies that there is a $\partial \Gamma$-valued random variable $Z_{\infty}(x)$, such that $Z_{n} \rightarrow Z_{\infty}(x)$ almost surely, if $Z_{0}=x$. Let $\nu_{x}$ be the distribution of $Z_{\infty}(x)$ on $\partial \Gamma$. Factoring through the first step of the random walk, the strong Markov property yields

$$
\nu_{x}=\sum_{y} P(x, y) \nu_{y} \text {. }
$$

Now let $\varphi$ be continuous on $\partial \Gamma$. We define

$$
h(x)=\int_{\partial \Gamma} \varphi d \nu_{x}
$$

and claim that this is the required prolongation. By (5.2), $h$ is harmonic. We have to show that $h\left(x_{n}\right) \rightarrow \varphi(\xi)$ if $\left(x_{n}\right)$ is a sequence in $X$ which tends to $\xi \in \partial \Gamma$ in the end topology.

Let $\varepsilon>0$. By continuity of $\varphi$, using Theorem 1 , there is a neighborhood

$$
N=C_{F}(\xi) \backslash U, \quad F \in \mathscr{F}(E), \quad U \in \mathscr{F}(X),
$$

of $\xi$ such that $|\varphi(\eta)-\varphi(\xi)|<\varepsilon$ for every $\eta \in N \cap \partial \Gamma\left(=C_{F}(\xi) \cap \partial \Gamma\right)$. We obtain

$$
|h(x)-\varphi(\xi)| \leq \int_{\partial \Gamma}|\varphi(\eta)-\varphi(\xi)| d \nu_{x}(\eta) \leq \varepsilon+2\|\varphi\|_{\infty} \nu_{x}(\partial \Gamma \backslash N) .
$$

Suppose that $x \in N$. Observe that $Z_{n}$, starting at $Z_{0}=x$, must pass through some vertex of $\delta F$ if it converges to $\partial \Gamma \backslash N$. Hence, we obtain

$$
\nu_{x}(\partial \Gamma \backslash N)=\sum_{y \in \delta F} a(x, y) \nu_{y}(\partial \Gamma \backslash N),
$$

where $a(x, y)=\operatorname{Pr}\left[Z_{n}\right.$ enters $\delta F$ at $\left.y \mid Z_{0}=x\right]$. Now, $a(x, y) \leq H(x, y) \leq$ $r_{y} G(x, o)$, where $r_{y}=1 / G(y, o)$. Writing $r=\sum_{y \in \delta F} r_{y}$, we get

$$
\nu_{x}(\partial \Gamma \backslash N) \leq r G(x, o) \text { for all } x \in N \text {. }
$$

If $\left(x_{n}\right)$ tends to $\xi$, then it eventually belongs to $N$ and leaves every finite set. As $G$ vanishes at infinity, $\nu_{x_{n}}(\partial \Gamma \backslash N)$ tends to zero, and the second term in (5.4) can be made arbitrarily small. This proves continuity of the extended function.

To our knowledge, this general and simple version of the solution of the Dirichlet problem is new even in the locally finite case. A special result can be found in [De].

Remark 2. A point $\xi \in \partial \Gamma$ is called regular for the Dirichlet problem, if for every continuous function $\varphi$ on $\partial \Gamma$, its harmonic extension (5.3) is continuous at $\xi$. With no change in the above proof, one obtains the following. 
If the Green kernel vanishes at $\xi$, that is, $\lim _{x \in \xi} G(x, o)=0$, then $\xi$ is regular for the Dirichlet problem. Furthermore, suppose that the support of the limiting distribution $\nu_{x}$ on $\partial \Gamma$ contains more than one point for some (and hence all) $x \in X$. Then the regular points for the Dirichlet problem with respect to the end compactification are precisely the points where the Green kernel vanishes.

By $[\mathrm{Hu}]$, the random walk also converges in the Martin topology to a limit random variable with values in $M$. With a slight abuse of the notation of the above proof, we now write $\nu_{x}$ for the limiting distribution on $M$ of the random walk starting at $x$. Given the base point $o$, the Poisson boundary is the pair $\left(\operatorname{Supp}\left(\nu_{o}\right), \nu_{o}\right)$, or briefly the set $\operatorname{Supp}\left(\nu_{o}\right) \subset M$. Usually, one is interested in its realization up to sets of measure zero. It is a model (the largest possible) for the points attained at infinity by the random walk starting at $o$. Furthermore, if $h$ is a bounded harmonic function, then (cf. [Ca, Proposition 3.2]) there is a unique $\varphi \in L^{\infty}\left(\nu_{o}\right)$ such that

$$
h(x)=\int_{\partial \Gamma} K(x, \cdot) \varphi d \nu_{o} .
$$

As a by product of the last theorem, we obtain the Poisson boundary in the case of a tree.

Corollary 1. If $\Gamma$ is a tree, and if the Green kernel vanishes at infinity, then $\operatorname{Supp}\left(\nu_{o}\right)=\partial \Gamma$ and $\nu_{o}\left(X^{*}\right)=0$, so that the Poisson boundary can be identified with $\Omega$. In particular, $\bar{\Omega}=\partial \Gamma$.

Proof. We first show that $\operatorname{Supp}\left(\nu_{o}\right)=\partial \Gamma$. Assume that there is $\xi \in \partial \Gamma$ and a neighborhood $N$ of $\xi$ such that $\nu_{o}(N)=0$. Then we can find a continuous function $\varphi$ on $\partial \Gamma$ such that $\varphi \equiv 1$ on $\partial \Gamma \backslash N$ and $\varphi(\xi)=0$. If $h$ is its continuous extension (5.3) according to Theorem 4, then $h$ satisfies (5.5), so that $h \equiv 1$. But $h$ is then discontinuous at $\xi$, a contradiction.

If a trajectory of $Z_{n}$ converges to $x^{*} \in X^{*}$ then by (4.1) it must visit $x$ infinitely often. Indeed, using the description of convergence preceding Theorem 2 , there must be infinitely many different first edges amongst the geodesics from $x$ to the $Z_{n}$. Between any two such $n$ there must be an $m$ for which $Z_{m}=x$. Hence, $\nu_{0}\left(\left\{x^{*}\right\}\right)=0$; otherwise transience is contradicted. As $X^{*}$ is countable, $\nu_{o}\left(X^{*}\right)=0$, so that we may identify the Poisson boundary with $\Omega$ up to a set of measure zero.

From the proof of Corollary 1, we see in particular that Theorem 4 does not hold without the assumption that $G$ vanishes at infinity: the last example of [PW1] is a transient nearest neighbor random walk on a locally finite tree with the following properties: (1) $G$ does not vanish at infinity, and (2) $\operatorname{Supp}\left(\nu_{o}\right) \subset$ $\partial \Gamma$ strictly. As above, we can construct continuous functions on $\partial \Gamma$ without continuous harmonic extension.

\section{EXAMPLES AND REMARKS}

The results of the preceding sections apply to some types of random walks on infinitely generated groups. If $\mathbf{G}$ is a group generated by the countable set $A$, then its Cayley graph with respect to $A$ has vertex set $X=\mathbf{G}$, and the unoriented edges are given by $E=\{[x, x a]: x \in \mathbf{G}, a \in A\}$. 
Example 1. The free group with infinitely many generators. Let $\mathbf{G}$ be generated freely by $A=\left\{a_{i}: i=1,2, \ldots\right\}$, with unit element $e$. Its Cayley graph with respect to $A$ is a tree, where all vertices have a countably infinite number of neighbors. The ends can be represented by infinite reduced words over $A \cup A^{-1}$, where the only reduction rules are $a_{i} a_{i}^{-1}=a_{i}^{-1} a_{i}=e$, and the set of improper vertices $\mathbf{G}^{*}$ is in one-to-one correspondence with $\mathbf{G}$.

If $p$ is a probability measure on $\mathbf{G}$, then it gives rise to a random walk on G with $P(x, y)=p\left(x^{-1} y\right)$. If $P$ is irreducible (i.e., $\operatorname{Supp}(p)$ generates $\mathbf{G}$ as a semigroup), then it is transient, and the Green kernel $G(\cdot, e)$ is in $l^{2}(\mathbf{G})$ [DG]. Hence, it vanishes at infinity. (We choose $e$ as our base point.)

If $\operatorname{Supp}(p)=A \cup A^{-1}$, then $P$ satisfies (4.1) with respect to the tree. All our results apply in this case: the Martin boundary is $\Omega \cup \mathbf{G}^{*}$, the minimal Martin boundary is $\Omega$, a Fatou theorem holds, the Dirichlet problem is solvable with respect to the Martin boundary, and the Poisson boundary charges all of $\Omega$.

Example 2. Free products of groups. Let $\mathbf{G}$ be the free product of infinitely many nontrivial, countable groups $\mathbf{G}_{i}, i=1,2, \ldots$, each one generated by a set $A_{i}$. The Cayley graph $\Gamma$ of $\mathbf{G}$ with respect to $A=\bigcup_{i} A_{i}$ is not a tree, but has tree-like features. Let $P$ arise as in Example 1 from a probability measure $p$ on $\mathbf{G}$ supported by $A \cup A^{-1}$.

If each $\mathbf{G}_{i}$ is finite, then the ends of $\Gamma$ can be identified with infinite words

$$
x_{i_{1}} x_{i_{2}} x_{i_{3}} \ldots, \quad \text { where } x_{i_{j}} \in \mathbf{G}_{i_{j}} \backslash\{e\} \text { and } i_{j+1} \neq i_{j} \text {. }
$$

In this setting, with some minor adaptations, all the results which we have stated for trees apply as in the case of free groups. Along the lines of [Wo], one can also describe the Martin boundary if the $\mathbf{G}_{i}$ are not assumed to be finite, but we omit the details.

Remark 3. Contrary to the finitely generated case (see, e.g., [De]), in the above examples it is crucial that the support of $p$ is $A \cup A^{-1}$, where $A$ is the described set of generators. Indeed, assume in the case of the free group, that $\operatorname{Supp}(p)$ is the set of words of length at most 2. The resulting random walk has property (4.1) with respect to the graph $\Gamma$ which we obtain from the tree by connecting every pair of vertices at distance at most 2 by an edge. But $\partial \Gamma$ has only one element, because $\Gamma \backslash F$ is connected for each $F \in \mathscr{F}(E)$. In fact, we do not know the Martin boundary in this case. However, we remark that Furstenberg [Fu] describes a random walk on an infinitely generated free group, whose Poisson boundary coincides with the set of ends of a homogeneous tree of finite degree. The support of the underlying probability $p$ is not $A \cup A^{-1}$, and $p$ is only given implicitly.

If $\mathbf{G}$ is a finitely generated group and $A$ and $B$ are two finite sets of generators, then the end compactifications of the corresponding Cayley graphs are canonically homeomorphic [Fr]. Similarly, it is not hard to show the following for not necessarily finitely generated groups: if $A$ and $B$ are two sets of generators of the group $\mathbf{G}$, then the end compactifications of the corresponding Cayley graphs are homeomorphic, if the symmetric difference of $A$ and $B$ is finite. In our example (words of length at most 2 versus words of length 1 ), this sufficient condition is violated.

Remark 4. We briefly explain the Martin compactification of [Do, KSK] and why we give preference to the one of $[\mathrm{Hu}, \mathrm{Re}]$. In [Do, KSK], the requirements 
are as follows: $b_{\mathscr{H}}(X)$ is the (up to canonical homeomorphism) unique compact space with the following properties:

(i' $) \quad X \subset b_{\mathscr{K}}(X)$, and $X$ is dense in $b_{\mathscr{K}}(X)$,

(ii' ) the kernels $K(x, \cdot), x \in X$, extend continuously to $b_{\mathscr{H}}(X)$, and

(iii' ${ }^{\prime}$ ) the extended kernels separate the points of $b_{\mathscr{H}}(X)$.

The resulting boundary is then $b_{\mathscr{H}}(X) \backslash X$. It suffices for all probabilistic purposes [KSK]. One can verify the following.

(a) There is a canonical surjection $\sigma: c_{\mathscr{H}}(X) \rightarrow b_{\mathscr{H}}(X)$,

(b) $\sigma$ is at most two-to-one,

(c) if $\sigma^{-1}(z)$ has two elements for $z \in b_{\mathscr{K}}(X)$, then $z=x \in X$, and

(d) in this case, the row $P(x, \cdot)$ of $x$ in the transition matrix must have infinitely many nonzero entries.

We omit the details. In particular, $c_{\mathscr{H}}(X)$ and $b_{\mathscr{H}}(X)$ coincide if there are only finitely many nonzero elements in each row of $P$.

Now let us consider a tree $\Gamma$, where every vertex has infinitely many neighbors. Let $P$ have property (4.1), with the Green kernel vanishing at infinity. Then the Dirichlet problem is solvable with respect to the Martin (三 end) compactification. Its not hard to verify that $b_{\mathscr{K}}(X)=X \cup \Omega$, and that $\sigma$ is given as follows: $\sigma(z)=z$, if $z \in X \cup \Omega$, and $\sigma\left(x^{*}\right)=x$, if $x^{*} \in X^{*}$. The topology on $b_{\mathscr{K}}(X)$ is the one inherited from $X^{*} \cup \Omega$ in $c_{\mathscr{K}}(X)$. In particular, $\Omega$ is dense in $b_{\mathscr{H}}(X)$. In this setting, the Dirichlet problem with respect to $b_{\mathscr{H}}(X) \backslash X=\Omega$ is not solvable.

\section{REFERENCES}

[Ca] P. Cartier, Fonctions harmoniques sur un arbre, Symposia Math. 9 (1972), 203-270.

[CS] D. I. Cartwright and S. Sawyer, The Martin boundary for general isotropic random walks in a tree, J. Theoret. Probab. 4 (1991), 111-136.

[CC] C. Constantinescu and A. Cornea, Ideale Ränder Riemannscher Flächen, Ergebnisse der Mathematik, Band 32, Springer-Verlag, Berlin, Göttingen and Heidelberg, 1963.

[De] Y. Derriennic, Marche aléatoire sur le group libre et frontière de Martin, Z. Wahrsch. Verw. Gebiete 32 (1975), 261-276.

[DG] Y. Derriennic and $Y$. Guivarc' $h$, Théorème de renouvellement pour les groupes non moyennables, C. R. Acad. Sci. Paris Sér. A 277 (1973), 613-615.

[Do] J. L. Doob, Discrete potential theory and boundaries, J. Math. Mech. 8 (1959), 433-458.

[Fr] H. Freudenthal, Über die Enden diskreter Räume und Gruppen, Comment. Math. Helv. 17 (1944), 1-38.

[Fu] H. Furstenberg, Random walks and discrete subgroups of Lie groups, Advances in Probability and Related Topics, Vol. 1 (P. Ney, ed.), Marcel Dekker, New York, 1971, pp. 1-63.

[Ha] R. Halin, Über unendliche Wege in Graphen, Math. Ann. 157 (1964), 125-137.

[Hu] G. A. Hunt, Markoff chains and Martin boundaries, Illinois J. Math. 4 (1960), 313-340.

[Ju] H. A. Jung, Connectivity in infinite graphs, Studies in Pure Math. (L. Mirsky, ed.), Academic Press, New York and London, 1971, pp. 137-143.

[Ke] J. L. Kelley, General topology, Graduate Texts in Math., vol. 27, Springer-Verlag, New York, Heidelberg and Berlin, 1975.

[KSK] J. G. Kemeny, J. L. Snell, and A. W. Knapp, Denumerable Markov chains, (2nd ed.). Graduate Texts in Math., vol. 40, Springer-Verlag, New York, Heidelberg and Berlin, 1976.

[Po] N. Polat, Aspects topologiques de la séparation dans les graphes infinis. I, Math. Z. 165 (1979), 73-100. 
[PW1] M. A. Picardello and W. Woess, Martin boundaries of random walks: ends of trees and groups, Trans. Amer. Math. Soc. 302 (1987), 185-205.

[PW2] $\frac{}{461 .}$. Harmonic functions and ends of graphs, Proc. Edinburgh Math. Soc. 31 (1988), 457-

[Re] D. Revuz, Markov chains, North-Holland, Amsterdam, 1975.

[Ta] J. C. Taylor, The Martin boundaries of equivalent sheaves, Ann. Inst. Fourier (Grenoble) 20 (1970), 433-456.

[Wo] W. Woess, $A$ description of the Martin boundary for nearest neighbor random walks on free products, Probability Measures on Groups. VIII (H. Heyer, ed.), Lecture Notes in Math., vol. 1210, Springer-Verlag, 1986.

School of Mathematics and Statistics, University of Sydney, N.S.W. 2006, Australia

Dipartimento di Matematica, Università di Milano, Via C. Saldini, 50, Milano 20133, ITALY 